\title{
Exosomes isolated from CAPS1-overexpressing colorectal cancer cells promote cell migration
}

\author{
BINGRUI WU ${ }^{1 *}$, DALONG SUN ${ }^{2,3^{*}}$, LIJIE MA $^{4}$, YIRAN DENG ${ }^{1}$, SI ZHANG $^{1}$, LING DONG $^{2}$ and SHE CHEN $^{1}$ \\ ${ }^{1}$ Key Laboratory of Glycoconjugate Research (Ministry of Public Health), Department of Biochemistry and Molecular Biology, \\ Shanghai Medical College, Fudan University; ${ }^{2}$ Department of Gastroenterology and Hepatology, Shanghai Institute of \\ Liver Diseases, Zhongshan Hospital, Fudan University, Shanghai 200032; ${ }^{3}$ Department of Gastroenterology and \\ Hepatology, Xiamen Branch, Zhongshan Hospital, Fudan University, Xiamen, Fujian 361006; ${ }^{4}$ Department of \\ Liver Surgery and Transplantation, Liver Cancer Institute, Zhongshan Hospital, Fudan University, \\ Key Laboratory of Carcinogenesis and Cancer Invasion of Ministry of Education, Shanghai 200032, P.R. China
}

Received May 10, 2019; Accepted September 6, 2019

DOI: $10.3892 /$ or.2019.7361

\begin{abstract}
Calcium-dependent activator protein for secretion 1 (CAPS1) has been reported to promote metastasis in colorectal cancer (CRC), however, the underlying mechanisms have not yet been elucidated. The present study revealed that exosomes derived from CAPS1-overexpressing CRC cells could enhance the migration of normal colonic epithelial FHC cells. GW4869, an inhibitor of exosomes, could attenuate the migration of FHC cells. Furthermore, liquid chromatography-mass spectrometry (LC-MS) and bioinformatics analysis demonstrated that overexpression of CAPS1 could alter the expression pattern of exosomal proteins involved in cell migration. Bone morphogenetic protein 4, which may serve vital roles in the process of CAPS1-induced cell migration, was downregulated in the exosomes. In summary, the present results demonstrated that CAPS1 promotes cell migration by regulating exosomes. Inhibiting the secretion of exosomes may be helpful for the treatment of patients with metastatic CRC.
\end{abstract}

Correspondence to: Professor Ling Dong, Department of Gastroenterology and Hepatology, Shanghai Institute of Liver Diseases, Zhongshan Hospital, Fudan University, 180 Fenglin Road, Xuhui, Shanghai 200032, P.R. China

E-mail: dong.ling@zs-hospital.sh.cn

Professor She Chen, Key Laboratory of Glycoconjugate Research (Ministry of Public Health), Department of Biochemistry and Molecular Biology, Shanghai Medical College, Fudan University, 130 Dong'an Road, Xuhui, Shanghai 200032, P.R. China

E-mail: shechen@fudan.edu.cn

*Contributed equally

Key words: CAPS1, exosome, colorectal cancer, liquid chromatography-mass spectrometry, proteomics, BMP4

\section{Introduction}

Colorectal cancer (CRC) is one of the most commonly diagnosed cancer types, and it accounts for $10 \%$ of all cancer cases, with an occurrence of $>1.8$ million cases every year worldwide (1). Treatment strategies involving surgery, chemotherapy and radiotherapy have increased the overall survival rates for patients with early stage CRC $(2,3)$. However, $60 \%$ of patients with CRC develop metastasis, which is the major cause of mortality in patients with CRC $(4,5)$. Thus, it is important to elucidate the mechanisms underlying the metastasis of CRC, and to investigate new CRC treatments based on the discoveries.

The activation of pro-oncogenes KRAS, $c-S r c$ and $c-M y c$, and inactivation of tumor suppressor genes, such as the loss of adenomatous polyposis coli $(A P C)$ and tumor suppressor p53 (TP53), all contribute to CRC tumorigenesis and development (2). Exosomes, as mediators of intercellular communication, have been reported to be involved in CRC progression (6,7). Exosomes are extracellular vesicles (EVs) with a diameter of $30-180 \mathrm{~nm}$, secreted by various cells (8-10). A recent study demonstrated that exosomes derived from the HCT-8 CRC cell line contributed to the EMT process via miR-210 (6). Exosomes derived from CRC cells (HT29, SW480 and SW620) were revealed to increase the migration and invasion capacities of colonic mesenchymal cells $(7,11)$. In addition, drug resistance during tumor treatment could also be attributed to exosomes. miR-145 and miR-34a released via exosomes are hypothesized to enhance the 5-fluorouracil resistance in DLD-1 cells (12). Epidermal growth factor receptor has been identified to be expressed on the surface of exosomes and was able to bind to Cetuximab, which reduced its therapeutic efficacy in Caco2 and HCT-116 cells (13).

Exosomes form as intraluminal vesicles (ILVs) by budding into early endosomes and multi-vesicular bodies (MVBs). Subsequently, MVBs are released upon fusion with the plasma membrane (PM) $(14,15)$. The SNARE complex may serve a role in the fusion of MVBs with the PM. However, the underlying mechanism remains to be fully elucidated (16). On the other hand, the intracellular adaptor protein syntenin, 
endosomal sorting complex required for transport (ESCRT), heparanase and tetraspanins demonstrate key roles in the loading of exosomes (7). In addition, sumoylation is considered to be a mechanism involved in the loading of miRNAs into exosomes (17).

$\mathrm{Ca}^{2+}$-dependent activator protein for secretion 1 (CAPS1) is characterized as a $145-\mathrm{kDa}$ brain protein containing a central pleckstrin homology domain, a Munc-13 homology (MH) domain, a C-terminal domain, a C-terminal membrane-association domain and a C2 domain (18). As a special regulator of the $\mathrm{Ca}^{2+}$-dependent large dense-core vesicle fusion process, CAPS1 promotes the assembly of the SNARE complex via the MH domain, which regulates vesicle exocytosis $(19,20)$. Our previous study demonstrated that CAPS1 was upregulated in $\mathrm{CRC}$, and that increased CAPS1 expression was associated with frequent metastasis and poor prognosis in patients with CRC (18). Furthermore, CAPS1 was revealed to promote CRC cell migration and invasion in vitro and facilitate CRC liver metastasis in vivo (18). However, whether exosomes are required for CAPS1-induced CRC metastasis requires further investigation.

BMP4 is a member of bone morphogenetic proteins (BMPs), which are multi-functional cytokines belonging to the transforming growth factor- $\beta$ (TGF- $\beta$ ) family (21). Previous studies suggest that BMP4 is closely associated with tumorigenesis $(22,23)$. In CRC, BMP4 was revealed to be frequently upregulated due to aberrant activation of Wnt- $\beta$-catenin signaling, and promoted cell migration and invasion $(22,24)$. Knockdown of BMP4 inhibited tumor formation of CRC cells in vivo through apoptosis induction (22). The expression of BMP4 was significantly increased in hepatocellular carcinoma (HCC) tissues (25). Increased BMP4 was correlated with high metastasis of HCC cells (25). BMP4 facilitated HCC cell invasion and metastasis though ID2-mediated EMT and promoted HCC cell proliferation via autophagy activation $(23,25)$. In breast cancer, BMP4 promoted cell migration and invasion possibly via induction of MMP-1 and CXCR4 expression (26). The function of BMP4 appears to be divergent but with clear evidence supporting tumor suppressing functions in lung squamous cell carcinoma (SQC). For example, decreased BMP4 induced by SOX2 enhanced lung SQC cell growth (27). This finding indicated a tissue context specific role of BMP4.

The present study revealed that CAPS1 promoted FHC cell migration by altering the protein expression profile of exosomes derived from CRC cells. GW4869, an exosome inhibitor, inhibited CAPS1-induced cell migration.

\section{Materials and methods}

Cell culture and conditioned medium preparation. The cell lines, HT29, SW480, FHC and 293T, used in the present study were purchased from The American Type Culture Collection. The cells were cultured in complete DMEM containing $10 \%$ fetal bovine serum (FBS; Gibco; Thermo Fisher Scientific, Inc.), $100 \mathrm{mg} / \mathrm{ml}$ penicillin, and $10 \mathrm{mg} / \mathrm{ml}$ streptomycin (Gibco; Thermo Fisher Scientific, Inc.), at $37^{\circ} \mathrm{C}$ and $5 \%$ $\mathrm{CO}_{2}$. Conditioned medium (CM) was harvested at $48 \mathrm{~h}$ from confluent cultures with exosome-depleted medium and centrifuged at $1,400 \mathrm{x} \mathrm{g}$ for $2 \mathrm{~min}$ at $4^{\circ} \mathrm{C}$ to remove cellular debris.
To inhibit exosome secretion, cells were treated with $10 \mu \mathrm{M}$ GW4869 (MedChemExpress) before collecting the CM.

Exosome isolation and characterization. Exosomes were isolated from HT29/SW480 CM by serial centrifugation. The medium was subjected to ultracentrifugation at $100,000 \mathrm{x} \mathrm{g}$ for $6 \mathrm{~h}$ at $4^{\circ} \mathrm{C}$ and washed with PBS $(100,000 \mathrm{x}$ g for $20 \mathrm{~min})(7,28)$. Subsequently, the exosomes were re-suspended in PBS. The presence of exosomes was confirmed by particle size with a Nanoparticle Tracking Analysis (NTA) system (NTA 3.2 Dev Build 3.2.16, Malvern Panalytical Ltd., Malvern, UK), and the expression of exosome-specific markers such as tumor susceptibility gene 101 protein (TSG101) and CD81 was evaluated by western blot analysis.

Electron microscopy. For electron microscopy, exosomes were fixed with $2 \%$ paraformaldehyde and loaded on carbon-coated copper grids. The grids were placed on $2 \%$ gelatin for $20 \mathrm{~min}$ at $37^{\circ} \mathrm{C}$ and washed with $0.15 \mathrm{M}$ glycine in PBS. Subsequently, the sections were blocked with $1 \%$ cold water fish-skin gelatin $(11,29,30)$. The grids were viewed under a Philips CM120 transmission electron microscope (Philips Research).

Exosome uptake assay. The exosomes were fluorescently labeled using an ExoGlow-Protein EV Labeling kit (System Biosciences), according to the manufacturer's instructions. Approximately 100-500 $\mu \mathrm{g}$ of labeled exosomes were added to $1 \times 10^{5} 293 \mathrm{~T} / \mathrm{FHC}$ cells. The red fluorescent signal was observed at 24 and $48 \mathrm{~h}$.

Cell migration assay. Transwell migration assays were performed in 24-well chambers (pore size, $0.8 \mu \mathrm{m}$; EMD Milipore). FHC cells were treated with HT29 cell-derived exosomes, HT29/SW480-CM or HT29/SW480-CM+GW4869 for $24 \mathrm{~h}$. FHC cells were then re-suspended in FBS-free DMEM and added to the upper chamber. DMEM containing $10 \%$ FBS was added to the lower chamber. The cells were incubated for 5 days, and subsequently, the cells on the undersurface of the membrane were fixed by $4 \%$ paraformaldehyde for $30 \mathrm{~min}$ at room temperature and stained with $0.1 \%$ crystal violet overnight at room temperature. The cells that had migrated through the filter were counted in four randomly selected regions per filter.

Western blot analysis. The cells or exosomes were homogenized in RIPA lysis buffer supplemented with protease inhibitors (Roche Diagnostics). The concentration of proteins was measured using the BCA Protein assay kit (Beyotime Institute of Biotechnology). Western blot analysis was performed as previously described (18). A total of $40 \mu \mathrm{g}$ proteins per lane were loaded. The cell or exosome lysates were immunoblotted with antibodies against CAPS1 (cat. no. ab32011; Abcam), $\beta$-actin (cat. no. ab8227), CD63 (cat. no. ab59479), CD81 (cat. no. ab35026), TSG101 (cat. no. ab133586; all from Abcam) or BMP4 (cat. no. A0425; ABclonal).

Quantitative proteomics and bioinformatics analyses of exosomal proteins. Proteins were extracted from the isolated exosomes, digested with trypsin, and analyzed by LC-MS. The 
mass spectra were searched against the Swiss-Prot Human Proteome database (https://www.uniprot.org/). Gene Ontology (GO) (http://www.omicsbean.com:88/) and Ingenuity Pathway Analysis (IPA) analysis software were used to analyze differentially expressed proteins.

Statistical analysis. The data are presented as the means \pm standard deviation. GraphPad Prism 7 software (GraphPad Prism, Inc.) was used for statistical analysis. Student's t-test was used for comparisons between groups. $\mathrm{P}<0.05$ (two-sided) was considered to indicate a statistically significant difference.

\section{Results}

Characterization of exosomes isolated from CAPS1-overexpressing CRC cells. Our previous study indicated that CAPS1 was upregulated in CRC, and that increased CAPS1 facilitated CRC metastasis (18). The previous study generated stable CAPS1-overexpressing HT29 and SW480 cell lines (CAPS1-HT29 and CAPS1-SW480) by lentiviral infection, as well as corresponding control cell lines (18). The protein level of CAPS1 was detected in CAPS1-HT29 and CAPS1-SW480 cells in the present study (Fig. 1A). In order to confirm the role of exosomes in the process of CAPS1-induced CRC metastasis, exosomes were isolated from the CM of CAPS1-overexpressing HT29 cells (CAPS1-HT29-CM) and control cells (Fig. 1B). The harvested exosomes were detected by the presence of exosome-specific markers, TSG101 and CD81, using western blot analysis (Fig. 1C). The morphology of the exosomes was characterized by transmission electron microscopy, which revealed no noticeable differences (Fig. 1D). The particles of exosomes were measured by NTA system, revealing a diameter range of 30-180 $\mathrm{nm}$. In comparison, exosomes derived from CAPS1-overexpressing cells exhibited a larger mean diameter. (Fig. 1E). To investigate whether HT29-derived exosomes (HT29-exo) could be internalized by target cells, the exosomes were pre-labeled fluorescently and co-cultured with 293T and FHC cells. Red fluorescence was observed within target cells at 24 and $48 \mathrm{~h}$, indicating that exosomes were endocytosed successfully by target cells (Fig. 1F).

Exosomes derived from CAPS1-overexpressing CRC cells enhance FHC migration. To investigate whether CAPS1 promotes CRC metastasis via exosomes. CAPS1-HT29-derived exosomes were co-cultured with FHC cells for $24 \mathrm{~h}$ (Fig. 2A). The FHC cell line is from normal fetal colonic mucosa, which exhibits a tumorigenic phenotype and has a metastatic potential in vivo $(31,32)$. A Transwell migration assay was performed to detect the migration of FHC cells, which demonstrated that exosomes derived from CAPS1-overexpressing CRC cells (HT29 and SW480) significantly enhanced the migration of FHC cells (HT29, P<0.001 SW480, P=0.0067; Fig. 2B).

To further investigate whether CRC cell-derived exosomes participate in FHC cell migration, GW4869, an inhibitor of exosomes, was used to inhibit exosome secretion in HT29 cells ( $\mathrm{P}=0.0038$; Fig. 2C). It was determined that GW4869 had no effect on HT29 cell viability ( $\mathrm{P}=0.4652$; Fig. $2 \mathrm{D})$. FHC cells were cultured with $\mathrm{CM}$ derived from CAPS1-overexpressing
CRC cells (HT29 and SW480), as well as control cells, for $24 \mathrm{~h}$. CM derived from CAPS1-overexpressing cells significantly increased FHC migration in the Transwell migration assay (HT29, P<0.001; SW480, P<0.001), an effect that was attenuated following GW4869-treatment (HT29, P=0.2275; SW480, $\mathrm{P}=0.227$; Fig. 2E), which indicated that CAPS1 promoted FHC cell migration via exosomes.

Proteomic profiling of exosomes derived from CAPS1-overexpressing CRC cells. The present study further characterized the changes in the exosome protein profile induced by CAPS1 overexpression (Fig. 3A). Proteins were extracted from exosomes derived from CAPS1-HT29 and control cells. LC-MS was applied to assess the proteomics of exosomes, and the raw LC-MS data files were searched against the Swiss-Prot Human Proteome database. A total of 1,024 proteins were identified and quantified (Table SI) (As aforementioned a total of 1035 proteins was identified, but only 1,024 proteins could be quantified since the intensity value of the remaining 11 proteins was extremely low and had to be ruled out for further analysis). CAPS1-HT29 and control cells had in total 437 exosomal proteins in common, while 138 exosomal proteins from CAPS1-HT29 differed from 135 proteins from control exosomes (Fig. 3B; Table SII). Further analysis revealed that 297 proteins were upregulated and 45 proteins were downregulated, with the cut-off threshold set at fold-change $=1.5$ and $\mathrm{P}<0.05$ (Fig. 3C; Table SIII).

GO enrichment analysis and the UniProt-GOA database were utilized to further classify the differentially expressed proteins (Fig. 3D). The enriched terms were cell adhesion, anchoring junction and cell adhesion binding for biological processes, cell components and molecular functions, respectively. Using IPA based on the Ingenuity Knowledge Base, p53 and CXCR3 were identified as the top upstream regulators (Fig. 3E)

According to the bioinformatics analysis, the candidate proteins that may participate in cell migration were identified (Fig. 4A). Previously, it has been reported that BMP4 is involved in metastasis in breast cancer, prostate cancer, $\mathrm{HCC}$ and CRC (24-26,33). Thus, BMP4 may serve vital roles in the process of CAPS1-induced cell migration. To investigate the variation of BMP4 in the exosomes derived from CAPS1-overexpressing CRC cells, western blot analysis was performed. It was revealed that BMP4 was downregulated $~ 0.5$-fold compared with Ctrl, which was consistent with the LC-MS results (Fig. 4B and C). These data indicated that exosomes are required for CAPS1-mediated cell migration (Fig. 4D).

\section{Discussion}

The present study demonstrated that exosomes derived from CAPS1-overexpressing CRC cells can enhance cell migration. Overexpression of CAPS1 led to alterations in the expression pattern of exosomal proteins, including the downregulation of BMP4, which was further confirmed.

CAPS1 is the vertebrate homologue of the Caenorhabditis elegans UNC-31 protein, which is involved in vesicle secretion (34). Previously, the essential role of CAPS1 in the development of cancer was investigated. Our previous study revealed that CAPS1 promoted CRC metastasis via the 

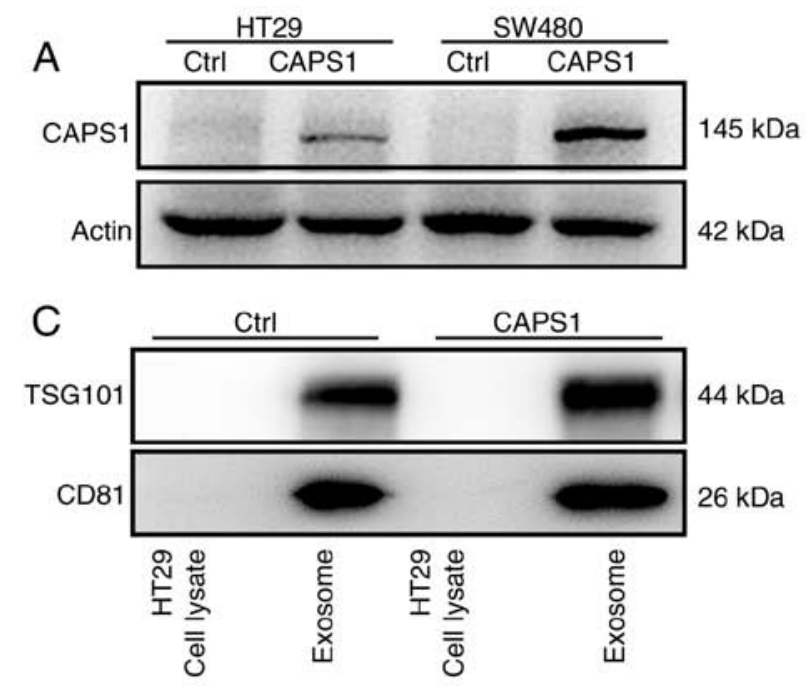

D Ctrl
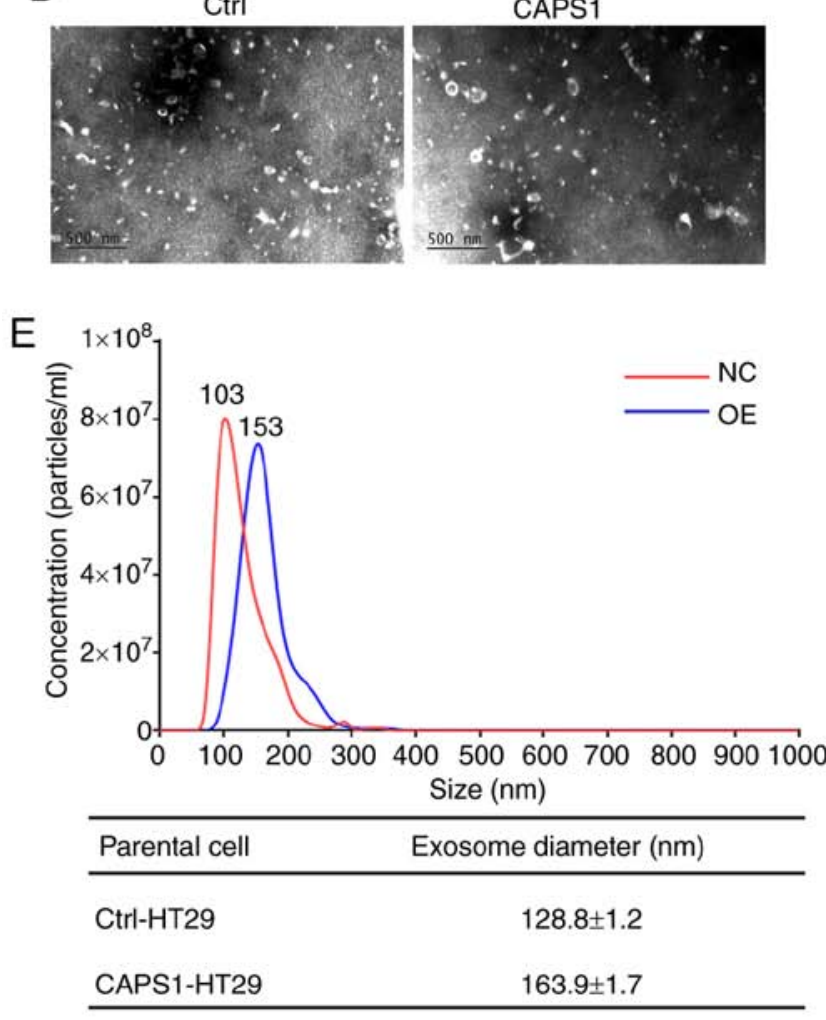
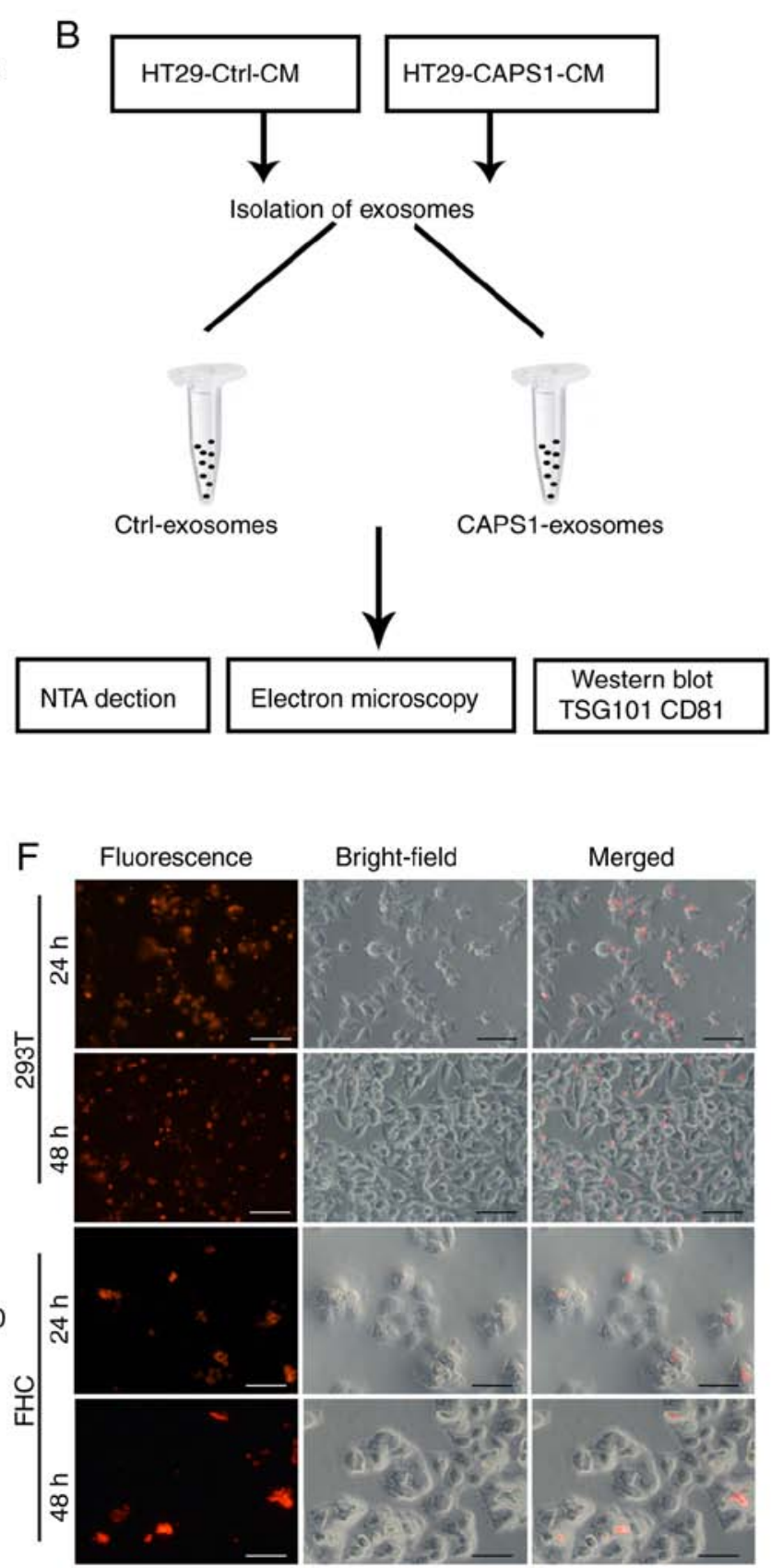

Figure 1. Characterization of exosomes isolated from CAPS1-overexpressing CRC cells. (A) The protein level of CAPS1was assessed in CAPS1-overexpressing CRC cells (HT29 and SW480) and normalized to the level of $\beta$-actin. (B) The workflow of exosome isolation and characterization. (C) The exosome markers TSG101 and CD81 were identified within the exosome fraction but not in the HT29 total cell fraction. (D) Electron micrograph of exosomes isolated from CAPS1-HT29 and control cells, revealing their typical morphology and size. Scale bar, $500 \mathrm{~nm}$. (E) The diameters of the exosomes isolated from CAPS1-HT29 and control cells were measured by the NTA system. (F) Exosomes isolated from HT29 cells were fluorescently pre-labeled and incubated with 293T and FHC cells. Images were captured at 24 and $48 \mathrm{~h}$ after the addition of exosomes. Scale bar, $50 \mu \mathrm{m}$. CAPS1, calcium-dependent activator protein for secretion 1; CRC, colorectal cancer; TSG101, tumor susceptibility gene 101 protein; NTA, Nanoparticle Tracking Analysis.

PI3K/Akt/GSK3 $\beta /$ Snail signaling pathway-mediated EMT process (18). In addition, it was demonstrated that CAPS1 serves as a biomarker of HCC, and the expression of CAPS1 is decreased in patients with aggressive HCC (35). Further investigation revealed that CAPS1 likely inhibits HCC development via alteration of the exocytosis-associated tumor microenvironment (36). In addition, decreased CAPS1 expression was revealed to be correlated with poor prognosis of patients with central nervous system primitive neuro-ectodermal tumors (37).
Metastasis is the predominant pathological reason for the mortality of patients with CRC. Notably, increasing evidence suggests that exosomes serve as key mediators in tumor metastasis $(11,38)$. It was reported that exosomes from highly liver metastatic CRC cell lines could significantly enhance the migration ability of Caco- 2 cells, a CRC cell line with poor liver metastatic potential (11). Similarly, the present study revealed that exosomes derived from CAPS1-overexpressing CRC cells facilitated FHC migration. The function of 
A

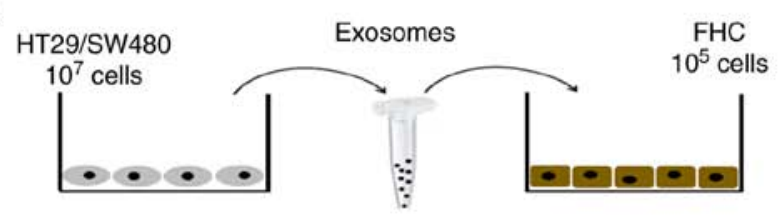

B

HT29-exo-FHC

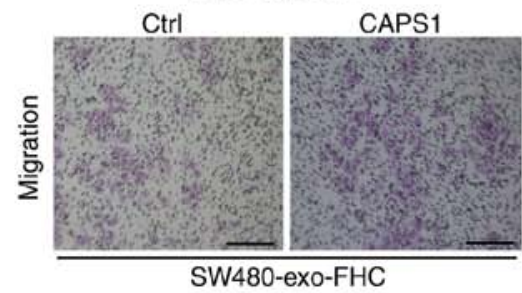

SW480-exo-FHC
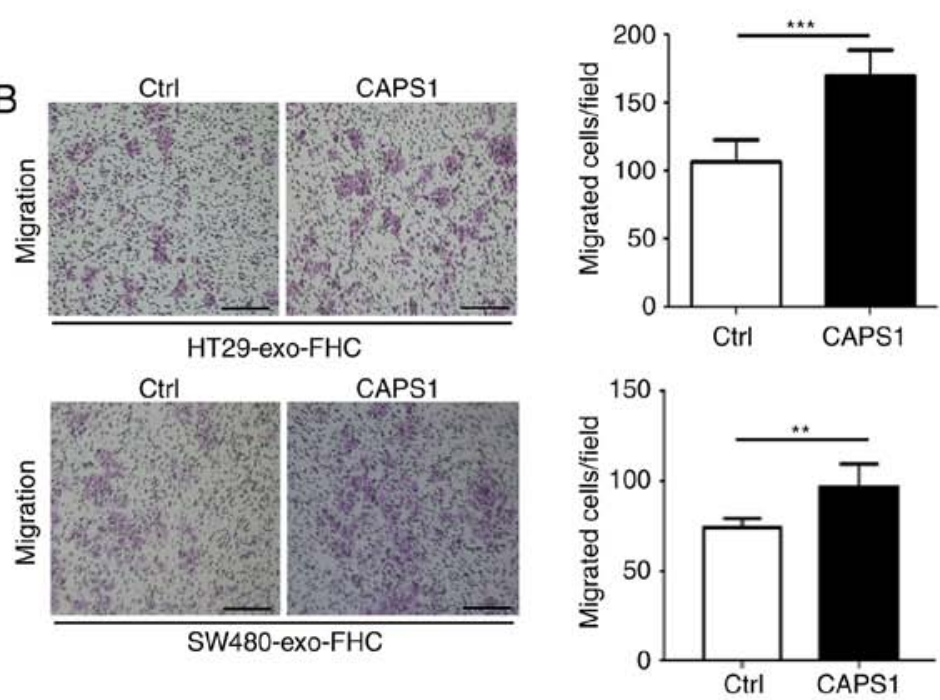

Ctrl

E

E

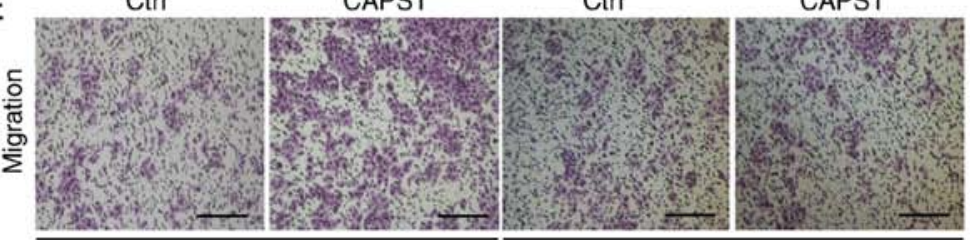

HT29-CM-FHC

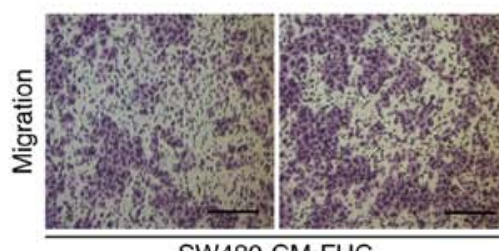

SW480-CM-FHC
HT29-CM+GW4869-FHC

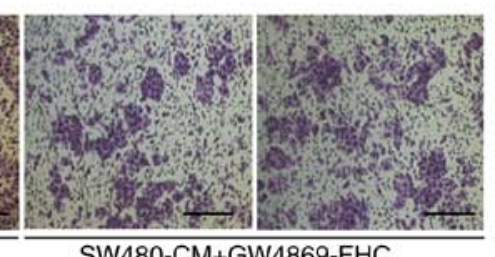

C

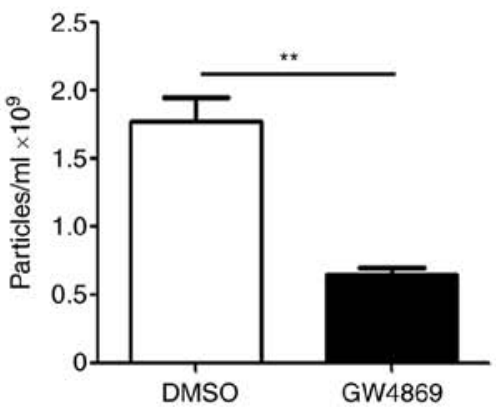

$\mathrm{D}$
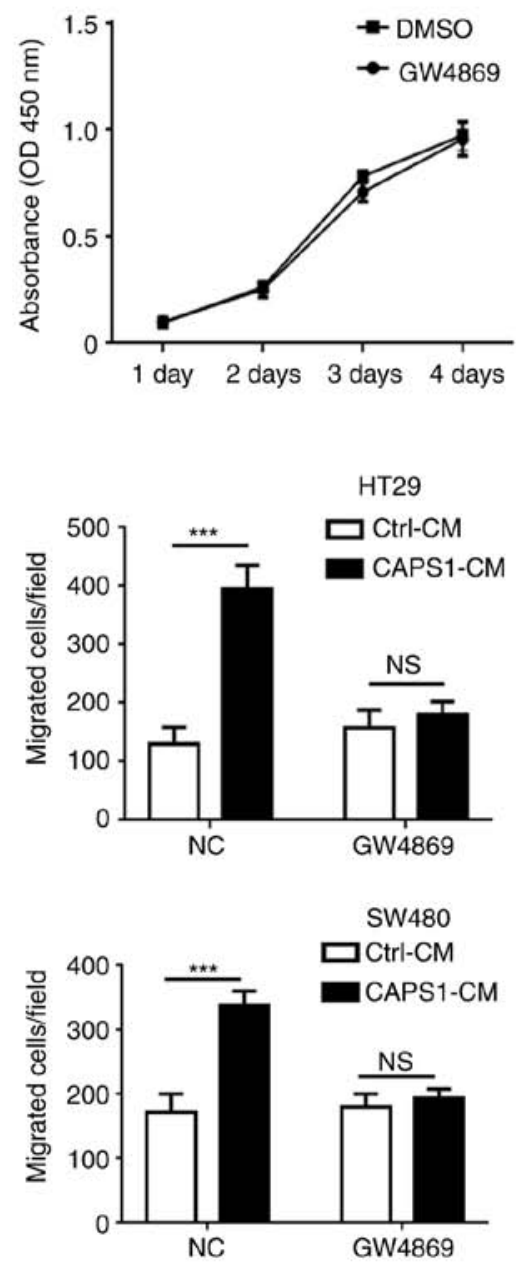

Figure 2. Exosomes from CAPS1-overexpressing CRC cells enhance FHC migration. (A) The workflow of FHC cells treated with HT29/SW480-derived exosomes. (B) Exosomes derived from CAPS1-HT29/SW480 promoted FHC migration as determined by a Transwell migration assay. (C) GW4869 inhibited the secretion of exosomes in HT29 cells. (D) The viability of HT29 cells pretreated with GW4869 was not affected in the CCK-8 assay. (E) GW4869 attenuated the migration of FHC cells. Scale bar, $50 \mu \mathrm{m}$ (left panel). The number of cells that migrated to the underside of the membrane was counted in four randomly selected regions per filter (right panel). Results of the Transwell migration assay are presented as the means \pm standard deviation $(\mathrm{n}=3)$. Magnification, $\mathrm{x} 100$. ${ }^{* *} \mathrm{P}<0.01$ and ${ }^{* * *} \mathrm{P}<0.001$. CAPS1, calcium-dependent activator protein for secretion 1 ; CRC, colorectal cancer.

exosomes in tumors predominantly depends on their communication between cells. For example, exosomes can transfer oncogenes, such as lncRNA H19 and miR-193a, to CRC, which promotes cancer progression $(39,40)$. Exosomes isolated from the serum of CRC patients were revealed to transfer malignant traits and confer the same phenotype of the primary tumor cells to the target cells (41). Exosomes, as a communication media for genetic exchange between cells, likely contribute to the process of metastasis mediated by CAPS1 in CRC.
CAPS1 is multi-domain protein. Among these domains, MH domain drives trans-SNARE complex formation and mediates membrane fusion through syntaxin interactions $(42,43)$. Notably, SNARE complex protein YKT6 has been identified to potentially be involved in the fusion of MVBs with the PM during the biogenesis of exosomes (16). Whether CAPS1 regulates the secretion and loading of exosomes via the SNARE complex in CRC requires further investigation.

Bioinformatics strategies have frequently been used to evaluate the function of exosomes $(35,44)$. In the present bioin- 
A

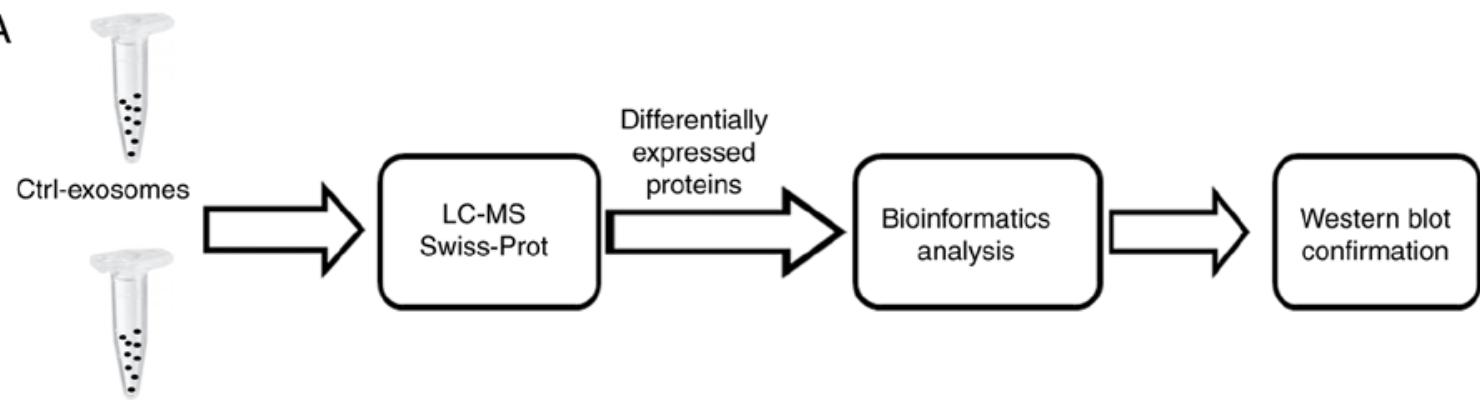

CAPS1-exosomes

B

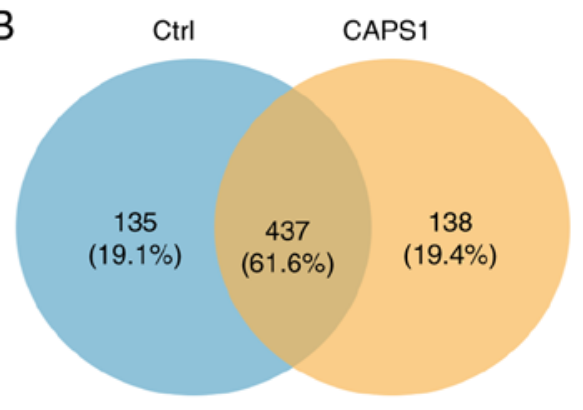

C

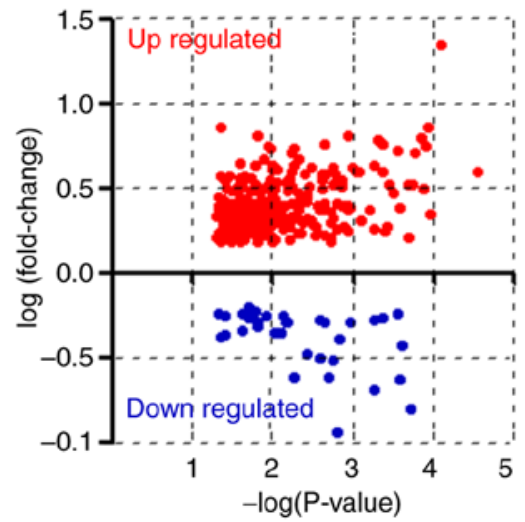

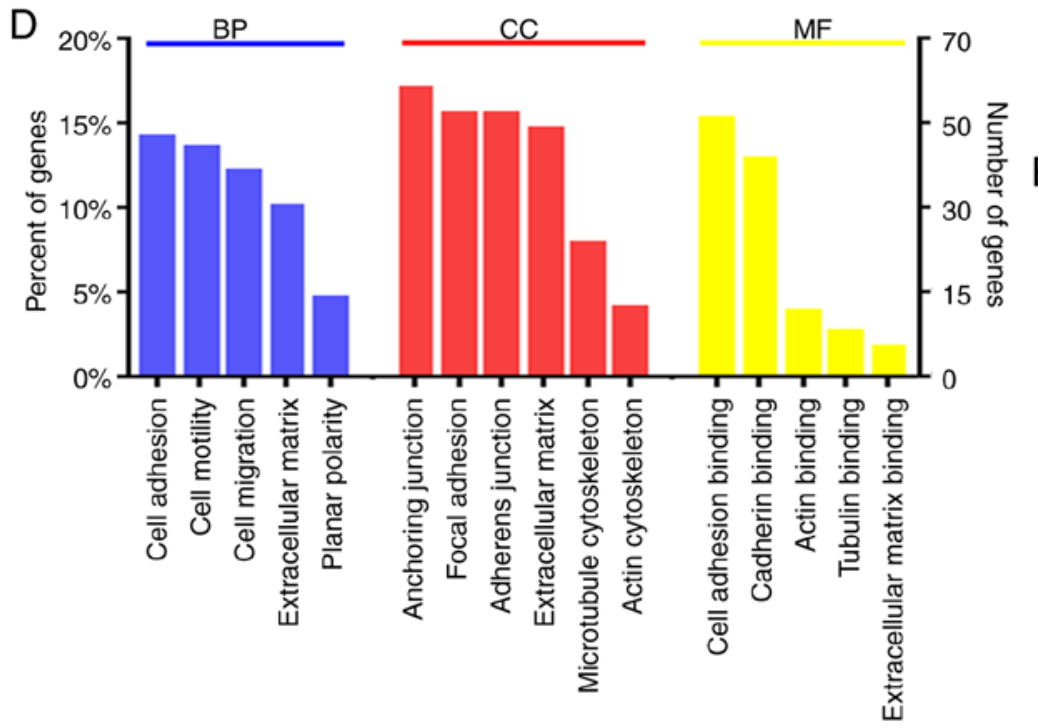

E

\begin{tabular}{ll} 
Top upstream regulators & \\
Upstream regulator & P-value \\
\hline TP53 & $4.36 \mathrm{e}-02$ \\
CXCR3 & $4.50 \mathrm{e}-02$
\end{tabular}

Figure 3. LC-MS and bioinformatics analysis of the differentially expressed exosomal proteins. (A) The workflow of LC-MS and bioinformatics analysis of differentially expressed exosomal proteins. (B) Venn diagram revealing the differentially expressed proteins identified in CAPS1-HT29 and control cell-derived exosomes. (C) The exosomal protein profile was altered by CAPS1-overexpression. In total, 297 proteins were significantly upregulated and 45 proteins were significantly downregulated. (D) GO analysis of differentially expressed exosomal proteins. (E) The upstream regulators of differentially expressed exosomal proteins in the IPA analysis. LC-MS, liquid chromatography-mass spectrometry; CAPS1, calcium-dependent activator protein for secretion 1; GO, Gene Ontology; IPA, Ingenuity Pathway Analysis.

formatics analysis, the differentially expressed exosomal proteins involved in MAPK, Wnt, TGF- $\beta$, PI3K-AKT, or RAS signaling, which were frequently dysregulated in CRC and were associated with CRC metastasis (45-50), were all considered as candidate proteins involved in cell migration induced by CAPS1 (Fig. 4A). In view of the role of BMP4 in metastasis, the present study examined the expression level of BMP4 in the exosomes, and the downregulation of BMP4 was further confirmed by western blot analysis
(Fig. 4B and C). However, the role of BMP4 in the process of CAPS1-induced cell migration should be further investigated. Furthermore, p53 and CXCR3 were identified as upstream regulators. p53 is activated by stress stimuli, and in turn, it governs a complex anti-proliferative transcriptional program to serve a tumor suppressive role $(51,52)$. However, TP53 is highly mutated and loses its function in CRC (53). CXCR3 is a G protein-coupled receptor that binds to chemokines to mediate leukocyte trafficking, integrin activation, 

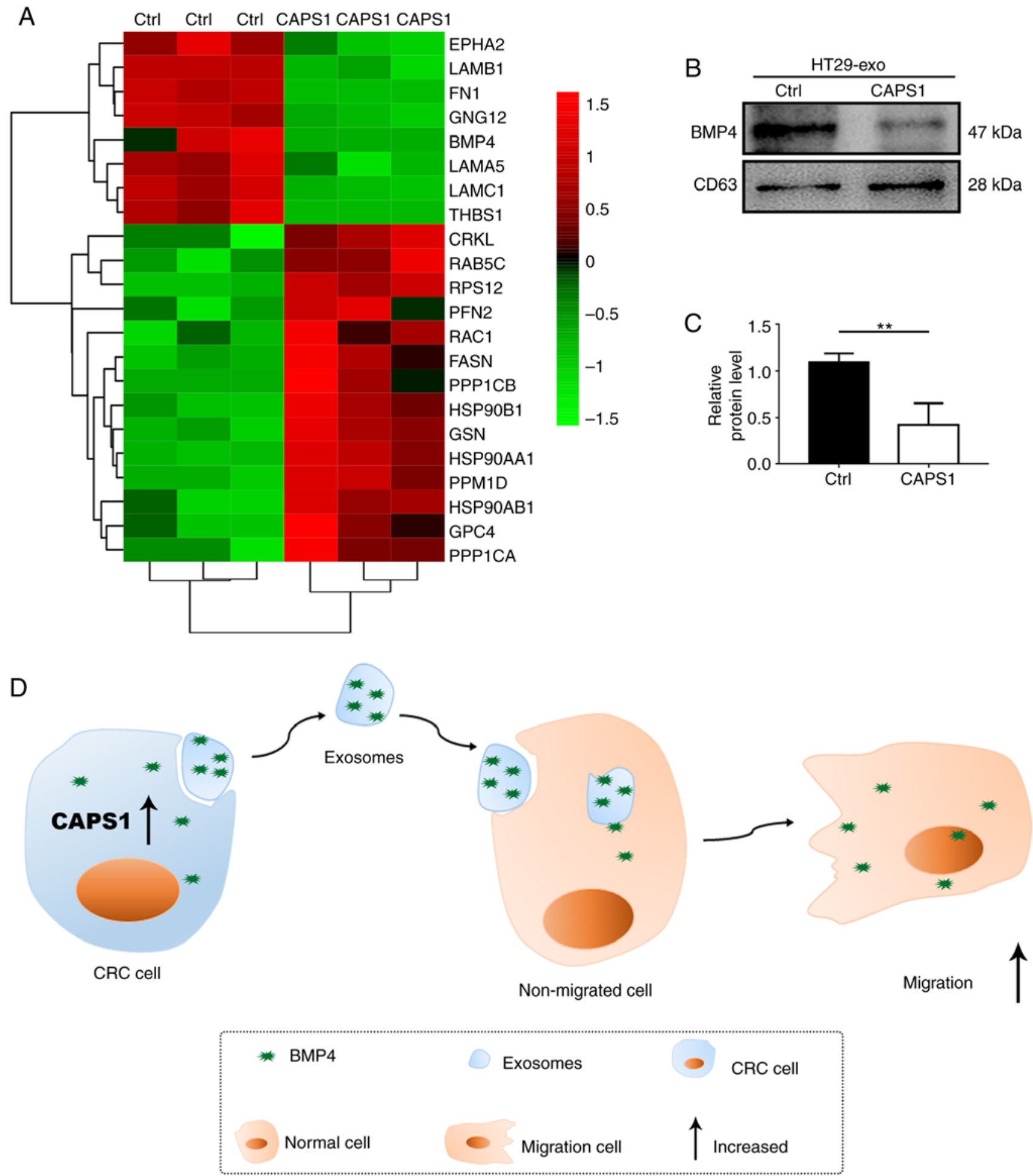

Figure 4. Candidate exosomal proteins that contribute to FHC cell migration. (A) Representative heatmap of the differentially expressed genes. The genes and samples are presented in the rows and columns, respectively. Red and green indicates upregulated and downregulated genes, respectively. The color scale bar indicates the $\log _{10}$ ratio of the intensities. (B) BMP4 protein level was assessed in exosomes derived from CAPS1-HT29 and control cells, and normalized to the level of CD63. (C) Quantification of the western blots was performed using ImageJ software and presented as the mean \pm standard deviation ( $\mathrm{n}=3$ ). ${ }^{* *} \mathrm{P}<0.01$. (D) A proposed working model for exosomes derived from CAPS1-overexpressing CRC cells in the promotion of cell migration. CAPS1, calcium-dependent activator protein for secretion 1; BMP4, bone morphogenetic protein 4.

cytoskeletal changes and chemotactic migration (54). A previous study reported that CXCR3 was upregulated in CRC and facilitated tumor metastasis via lymph nodes (55). Fibronectin 1 (FN1), which was downregulated in the present LC-MS analysis, is a target molecule of CXCR3.
Furthermore, FN1 has been revealed to regulate numerous molecules that are involved in cytoskeletal organization and integrin signaling during tumor progression and metastasis (56). Thrombospondin-1 (THBS-1), a target gene of $\mathrm{p} 53$, is an endogenous inhibitor of angiogenesis and was also 
downregulated in the current LC-MS analysis (57). BMP4, FN1 and THBS-1 transferred by exosomes may serve an essential role in the process of CAPS1-induced CRC cell migration.

CAPS1 had an effect on the diameter of the exosomes according to the analysis of mean diameter by NTA detection. The exosomes derived from CAPS1-HT29 cells exhibited a higher mean diameter. Similarly, the majority of CAPS1-HT29-derived exosomes presented with a diameter of $153 \mathrm{~nm}$, whereas those from control cells exhibited a diameter of $103 \mathrm{~nm}$ (Fig. 1E).

In summary, the present study demonstrated that CAPS1 promoted cell migration by remodeling the protein profile of exosomes. Furthermore, several candidate proteins were identified that may participate in the process of CRC metastasis mediated by CAPS1. However, the RNA profile of CAPS1-CRC-derived exosomes, and the proteomics and transcriptomic profiles of target cells require further analysis. The present data may improve understanding of a new target for the treatment of patients with CRC metastasis via the inhibition of exosome secretion.

\section{Acknowledgements}

We thank Dr Xiaoxiao Wang and Dr Yaohua Li from Key Laboratory of Glycoconjugate Research (Ministry of Public Health), Department of Biochemistry and Molecular Biology, Shanghai Medical college, Fudan University for technical assistance.

\section{Funding}

The present study was supported by grants from the National Natural Science Foundation of China (grant nos. 81772615 , $81672334,81573423,81770137$ and 81802302) and the Shanghai Science and Technology Commission (grant no. 15410710100).

\section{Availability of data and materials}

All data generated or analyzed during the present study are included in this published article.

\section{Authors' contributions}

BW, DS, LD and SC designed and conceived this project. BW, DS and LM developed methodology; BW, DS and YD performed experiments and generated data; SC, SZ analyzed and interpreted data; SC, BW wrote the manuscript. All authors contributed to and approved the manuscript.

\section{Ethics approval and consent to participate}

Not applicable.

\section{Patient consent for publication}

Not applicable.

\section{Competing interests}

There authors declare that they have no competing interests.

\section{References}

1. Bray F, Ferlay J, Soerjomataram I, Siegel RL, Torre LA and Jemal A: Global cancer statistics 2018: GLOBOCAN estimates of incidence and mortality worldwide for 36 cancers in 185 countries. CA Cancer J Clin 68: 394-424, 2018.

2. Mundade R, Imperiale TF, Prabhu L, Loehrer PJ and Lu T: Genetic pathways, prevention, and treatment of sporadic colorectal cancer. Oncoscience 1: 400-406, 2014.

3. Brody H: Colorectal cancer. Nature 521: S1, 2015.

4. Xu F, Tang B, Jin T and Dai C: Current status of surgical treatment of colorectal liver metastases. World J Clin Cases 6: 716-734, 2018.

5. Siegel RL, Miller KD and Jemal A: Cancer statistics, 2018. CA Cancer J Clin 68: 7-30, 2018.

6. Bigagli E, Luceri C, Guasti D and Cinci L: Exosomes secreted from human colon cancer cells influence the adhesion of neighboring metastatic cells: Role of microRNA-210. Cancer Biol Ther 17: 1062-1069, 2016.

7. Ruiz-López L, Blancas I, Garrido JM, Mut-Salud N, Moya-Jódar M, Osuna A and Rodríguez-Serrano F: The role of exosomes on colorectal cancer: A review. J Gastroenterol Hepatol 33: 792-799, 2018.

8. Kreimer S, Belov AM, Ghiran I, Murthy SK, Frank DA and Ivanov AR: Mass-spectrometry-based molecular characterization of extracellular vesicles: Lipidomics and proteomics. J Proteome Res 14: 2367-2384, 2015.

9. van Niel G, Porto-Carreiro I, Simoes S and Raposo G: Exosomes: A common pathway for a specialized function. J Biochem 140: 13-21, 2006.

10. Schorey JS and Bhatnagar S: Exosome function: From tumor immunology to pathogen biology. Traffic 9: 871-881, 2008.

11. Wang X, Ding X, Nan L, Wang Y, Wang J, Yan Z, Zhang W, Sun J, Zhu W, Ni B, et al: Investigation of the roles of exosomes in colorectal cancer liver metastasis. Oncol Rep 33: 2445-2453, 2015.

12. Akao Y, Khoo F, Kumazaki M, Shinohara H, Miki K and Yamada N: Extracellular disposal of tumor-suppressor miRs-145 and $-34 \mathrm{a}$ via microvesicles and 5-FU resistance of human colon cancer cells. Int J Mol Sci 15: 1392-1401, 2014.

13. Ragusa M, Statello L, Maugeri M, Barbagallo C, Passanisi R, Alhamdani MS, Li Destri G, Cappellani A, Barbagallo D, Scalia M, et al: Highly skewed distribution of miRNAs and proteins between colorectal cancer cells and their exosomes following Cetuximab treatment: Biomolecular, genetic and translational implications. Oncoscience 1: 132-157, 2014.

14. Kowal J, Tkach M and Théry C: Biogenesis and secretion of exosomes. Curr Opin Cell Biol 29:116-25, 2014.

15. Colombo M, Raposo G and Thery C: Biogenesis, secretion, and intercellular interactions of exosomes and other extracellular vesicles. Annu Rev Cell Dev Biol 30: 255-289, 2014.

16. Gross JC, Chaudhary V, Bartscherer K and Boutros M: Active Wnt proteins are secreted on exosomes. Nat Cell Biol 14: 1036-1045, 2012.

17. Villarroya-Beltri C, Gutiérrez-Vázquez C, Sánchez-Cabo F, Pérez-Hernández D, Vázquez J, Martin-Cofreces N, Martinez-Herrera DJ, Pascual-Montano A, Mittelbrunn M and Sánchez-Madrid F: Sumoylated hnRNPA2B1 controls the sorting of miRNAs into exosomes through binding to specific motifs. Nat Commun 4: 2980, 2013.

18. Zhao GX, Xu YY, Weng SQ, Zhang S, Chen Y, Shen XZ, Dong L and Chen S: CAPS1 promotes colorectal cancer metastasis via Snail mediated epithelial mesenchymal transformation. Oncogene 38: 4574-4589, 2019.

19. Speidel D, Varoqueaux F, Enk C, Nojiri M, Grishanin RN, Martin TF, Hofmann K, Brose N and Reim K: A family of $\mathrm{Ca} 2+-$ dependent activator proteins for secretion: Comparative analysis of structure, expression, localization, and function. J Biol Chem 278: 52802-52809, 2003.

20. Grishanin RN, Kowalchyk JA, Klenchin VA, Ann K, Earles CA, Chapman ER, Gerona RR and Martin TF: CAPS acts at a prefusion step in dense-core vesicle exocytosis as a PIP2 binding protein. Neuron 43: 551-562, 2004.

21. Ehata S, Yokoyama Y, Takahashi K and Miyazono K: Bi-directional roles of bone morphogenetic proteins in cancer: Another molecular Jekyll and Hyde? Pathol Int 63: 287-296, 2013.

22. Yokoyama Y, Watanabe T, Tamura Y, Hashizume Y, Miyazono K and Ehata S: Autocrine BMP-4 signaling is a therapeutic target in colorectal cancer. Cancer Res 77: 4026-4038, 2017. 
23. Deng G, Zeng S, Qu Y, Luo Q, Guo C, Yin L, Han Y, Li Y, Cai C, $\mathrm{Fu}$ Y, and Shen H: BMP4 promotes hepatocellular carcinoma proliferation by autophagy activation through JNK1-mediated Bcl-2 phosphorylation. J Exp Clin Canc Res 37: 156, 2018.

24. Zhou J, Liu H, Zhang L, Liu X, Zhang C, Wang Y, He Q, Zhang Y, Li Y, Chen Q, et al: DJ-1 promotes colorectal cancer progression through activating PLAGL2/Wnt/BMP4 axis. Cell Death Dis 9: $865,2018$.

25. Zeng S, Zhang Y, Ma J, Deng G, Qu Y, Guo C, Han Y, Yin L, Cai C, Li Y, et al: BMP4 promotes metastasis of hepatocellular carcinoma by an induction of epithelial-mesenchymal transition via upregulating ID2. Cancer Lett 390: 67-76, 2017.

26. Guo D, Huang J and Gong J: Bone morphogenetic protein 4 (BMP4) is required for migration and invasion of breast cancer. Mol Cell Biochem 363: 179-190, 2012.

27. Fang WT, Fan CC, Li SM, Jang TH, Lin HP, Shih NY, Chen CH, Wang TY, Huang SF, Lee AY, et al: Downregulation of a putative tumor suppressor BMP4 by SOX2 promotes growth of lung squamous cell carcinoma. Int J Cancer 135: 809-819, 2014

28. Ying W, Riopel M, Bandyopadhyay G, Dong Y, Birmingham A, Seo JB, Ofrecio JM, Wollam J, Hernandez-Carretero A, $\mathrm{Fu} \mathrm{W}$, et al: Adipose tissue macrophage-derived exosomal miRNAs can modulate in vivo and in vitro insulin sensitivity. Cell 171: 372-384.e12, 2017.

29. Li XJ, Ren ZJ, Tang JH and Yu Q: Exosomal MicroRNA MiR-1246 promotes cell proliferation, invasion and drug resistance by targeting CCNG2 in breast cancer. Cell Physiol Biochem 44: 1741-1748, 2017.

30. Singh R, Pochampally R, Watabe K, Lu Z and Mo YY: Exosome-mediated transfer of miR-10b promotes cell invasion in breast cancer. Mol Cancer 13: 256, 2014.

31. Soucek K, Gajdusková P, Brázdová M, Hýzd'alová $M$, Kocí L, Vydra D, Trojanec R, Pernicová Z, Lentvorská L, Hajdúch M, et al: Fetal colon cell line FHC exhibits tumorigenic phenotype, complex karyotype, and TP53 gene mutation. Cancer Genet Cytogenet 197: 107-116, 2010.

32. Siddiqui KM and Chopra DP: Primary and long term epithelial cell cultures from human fetal normal colonic mucosa. In Vitro 20: 859-868, 1984

33. Lin SC, Lee YC, Yu G, Cheng CJ, Zhou X, Chu K, Murshed M, Le NT, Baseler L, Abe JI, et al: Endothelial-to-osteoblast conversion generates osteoblastic metastasis of prostate cancer. Dev Cell 41: 467-480.e3, 2017.

34. Ann K, Kowalchyk JA, Loyet KM and Martin TF: Nove Ca2+-binding protein (CAPS) related to UNC-31 required for Ca2+-activated exocytosis. J Biol Chem 272: 19637-19640, 1997.

35. Liu T, Xue R, Huang X, Zhang D, Dong L, Wu H and Shen X: Proteomic profiling of hepatitis B virus-related hepatocellular carcinoma with magnetic bead-based matrix-assisted laser desorption/ionization time-of-flight mass spectrometry. Acta Biochim Biophys Sin (Shanghai) 43: 542-550, 2011.

36. Xue R, Tang W, Dong P, Weng S, Ma L, Chen S, Liu T, Shen X, Huang X, Zhang S, et al: CAPS1 negatively regulates hepatocellular carcinoma development through alteration of exocytosis-associated tumor microenvironment. Int J Mol Sci 17: pii: E1626, 2016

37. Miller S, Rogers HA, Lyon P, Rand V, Adamowicz-Brice M, Clifford SC, Hayden JT, Dyer S, Pfister S, Korshunov A, et al: Genome-wide molecular characterization of central nervous system primitive neuroectodermal tumor and pineoblastoma. Neuro Oncol 13: 866-879, 2011

38. Fang JH, Zhang ZJ, Shang LR, Luo YW, Lin YF, Yuan Y and Zhuang SM: Hepatoma cell-secreted exosomal microRNA-103 increases vascular permeability and promotes metastasis by targeting junction proteins. Hepatology 68: 1459-1475, 2018.

39. Ren J, Ding L, Zhang D, Shi G, Xu Q, Shen S, Wang Y, Wang T and Hou Y: Carcinoma-associated fibroblasts promote the stemness and chemoresistance of colorectal cancer by transferring exosomal lncRNA H19. Theranostics 8: 3932-3948, 2018.

40. Teng Y, Ren Y, Hu X, Mu J, Samykutty A, Zhuang X, Deng Z Kumar A,Zhang L,Merchant ML, et al: MVP-mediated exosomal sorting of miR-193a promotes colon cancer progression. Nat Commun 8: 14448, 2017
41. Abdouh M, Hamam D, Gao Z, Arena V, Arena M and Arena GO Exosomes isolated from cancer patients' sera transfer malignant traits and confer the same phenotype of primary tumors to oncosuppressor-mutated cells. J Exp Clin Canc Res 36: 113 2017.

42. James DJ, Kowalchyk J, Daily N, Petrie M and Martin TF: CAPS drives trans-SNARE complex formation and membrane fusion through syntaxin interactions. Proc Natl Acad Sci USA 106: $17308-17313,2009$.

43. Koch H, Hofmann $\mathrm{K}$ and Brose N: Definition of Munc13-homology-domains and characterization of a novel ubiquitously expressed Munc13 isoform. Biochem J 349: 247-253, 2000.

44. Ji H, Greening DW, Barnes TW, Lim JW, Tauro BJ, Rai A, Xu R, Adda C, Mathivanan S, Zhao W, et al: Proteome profiling of exosomes derived from human primary and metastatic colorectal cancer cells reveal differential expression of key metastatic factors and signal transduction components. Proteomics 13: $1672-1686,2013$

45. Fang YJ, Lu ZH, Wang GQ, Pan ZZ, Zhou ZW, Yun JP, Zhang MF and Wan DS: Elevated expressions of MMP7, TROP2, and survivin are associated with survival, disease recurrence, and liver metastasis of colon cancer. Int J Colorectal Dis 24: 875-884, 2009.

46. Urosevic J, Nebreda AR and Gomis RR: MAPK signaling control of colon cancer metastasis. Cell Cycle 13: 2641-2642, 2014.

47. Jiang Y, Liu XQ, Rajput A, Geng L, Ongchin M, Zeng Q, Taylor GS and Wang J: Phosphatase PRL-3 is a direct regulatory target of TGFbeta in colon cancer metastasis. Cancer Res 71: 234-244, 2011

48. Pancione M, Forte N,Fucci A, Sabatino L, Febbraro A, Di Blasi A, Daniele B, Parente D and Colantuoni V: Prognostic role of beta-catenin and p53 expression in the metastatic progression of sporadic colorectal cancer. Hum Pathol 41: 867-876, 2010.

49. Song $\mathrm{G}, \mathrm{Xu} \mathrm{S}$, Zhang $\mathrm{H}$, Wang $\mathrm{Y}$, Xiao C, Jiang T, Wu L, Zhang T, Sun X, Zhong L, et al: TIMP1 is a prognostic marker for the progression and metastasis of colon cancer through FAK-PI3K/AKT and MAPK pathway. J Exp Clin Canc Res 35: 148,2016

50. Bahrami A, Hassanian SM, ShahidSales S, Farjami Z, Hasanzadeh M, Anvari K, Aledavood A, Maftouh M, Ferns GA, Khazaei $\mathrm{M}$ and Avan A: Targeting RAS signaling pathway as a potential therapeutic target in the treatment of colorectal cancer. J Cell Physiol 233: 2058-2066, 2018.

51. Kastenhuber ER and Lowe SW: Putting p53 in Context. Cell 170 1062-1078, 2017.

52. Mills KD: Tumor suppression: Putting p53 in context. Cell Cycle 12: 3461-3462, 2013.

53. Iacopetta B: TP53 mutation in colorectal cancer. Hum Mutat 21: 271-276, 2003

54. Ma B, Khazali A and Wells A: CXCR3 in carcinoma progression. Histol Histopathol 30: 781-792, 2015.

55. Kawada K, Hosogi H, Sonoshita M, Sakashita H, Manabe T, Shimahara Y, Sakai Y, Takabayashi A, Oshima M and Taketo MM: Chemokine receptor CXCR3 promotes colon cancer metastasis to lymph nodes. Oncogene 26: 4679-4688, 2007.

56. Chen Y, Xie Y, Xu L, Zhan S, Xiao Y, Gao Y, Wu B and Ge W: Protein content and functional characteristics of serum-purified exosomes from patients with colorectal cancer revealed by quantitative proteomics. Int J Cancer 140: 900-913, 2017.

57. Jia L and Waxman DJ: Thrombospondin-1 and pigment epithelium-derived factor enhance responsiveness of KM12 colon tumor to metronomic cyclophosphamide but have disparate effects on tumor metastasis. Cancer Lett 330: 241-249, 2013.

This work is licensed under a Creative Commons Attribution-NonCommercial-NoDerivatives 4.0 International (CC BY-NC-ND 4.0) License. 